\title{
High-Risk PML Patients Switching from Natalizumab to Alemtuzumab: an Observational Study
}

\author{
Simona Malucchi · Marco Capobianco · Marianna Lo Re • \\ Maria Malentacchi · Alessia di Sapio · Manuela Matta • \\ Francesca Sperli • Antonio Bertolotto
}

Received: October 18, 2016 / Published online: December 3, 2016

(C) The Author(s) 2016. This article is published with open access at Springerlink.com

\begin{abstract}
Introduction: The choice of therapy in patients withdrawing from natalizumab treatment is still an open question and neurologists need strategies to manage this group of patients. The aim of this study is to evaluate if alemtuzumab is able to control the disease when used in patient who have stopped natalizumab.
\end{abstract}

Methods: 16 patients stopped natalizumab treatment after a median number of 20 infusions (range 12-114); all the patients were responders to natalizumab (neither clinical nor radiological activity during natalizumab therapy) and the reason for stopping was the risk of PML for all of them. Patients were switched to alemtuzumab after a median wash-out period of 70 days (range 41-99 days); patients underwent brain MRI every three

Enhanced content To view enhanced content for this article go to http://www.medengine.com/Redeem/ 0527F0603D472800.

S. Malucchi $(\varangle) \cdot$ M. Capobianco $\cdot$ M. Lo Re .

M. Malentacchi - A. di Sapio - M. Matta - F. Sperli .

A. Bertolotto

University Hospital San Luigi Gonzaga, SCDO

Neurologia 2-Regional Multiple Sclerosis Center,

Regione Gonzole 10, 10043 Orbassano, TO, Italy

e-mail: simona.malucchi@gmail.com months during natalizumab treatment and then just before starting alemtuzumab in order to exclude signs suggestive of PML; then, contrast-enhanced brain MRI was planned 6 and 12 months after alemtuzumab infusion.

Results: At present, 8 out of 16 patients have a follow-up $>6$ months and 2 out of 8 reached 1-year follow-up; 5 have a follow-up of $3-6$ months and 3 have a follow-up $<3$ months. Brain MRI at 6 months after alemtuzumab is available for 8 out of 16 patients and in all of them, neither signs of disease activity nor new lesions are present; in 2 out of 8 patients, brain MRI at 12 months is also available, showing no sign of disease activity. Clinical evaluation performed at 6 and at 12 months (when available) showed stability, in particular neither relapses nor increase in EDSS were observed.

Conclusions: Alemtuzumab was able to control the disease course in patients who stopped natalizumab; of course, as this is a single-centre study and the number of patients is small, these findings are very preliminary and need further confirmation.

Keywords: Alemtuzumab; Multiple Sclerosis; Natalizumab; PML 


\section{INTRODUCTION}

Alemtuzumab was approved by the EMA in 2013 for active relapsing-remitting multiple sclerosis (RRMS) patients. It is considered an induction treatment, since it causes lymphocyte depletion followed by cellular repopulation [1]. For this reason, the ideal candidate is an active patient in an early phase of the disease. In the real world, alemtuzumab is also used as a "third line" therapy, when many prior treatments have failed.

At present, neurologists also have other highly active drugs [2], but there are no head-to-head studies directly comparing the efficacy of alemtuzumab with other efficacious therapies such as natalizumab or fingolimod. Therefore, the decision to choose the most suitable medication largely depends on other factors, such as the potential side effects.

Patients with long-term natalizumab exposure and anti-JCV seropositivity who stop natalizumab for the risk of PML are a category of patients for whom no specific therapeutic strategy has been established. For this category, management is largely customized for the individual patient and alemtuzumab is an option, although no published data are available.

In this study we present our data showing that alemtuzumab is able to control disease course in a group of 16 patients previously treated with natalizumab and with an aggressive disease course before starting natalizumab.

\section{METHODS}

This is an observational study. The study used a sample of 16 RRMS patients with a median EDSS of 2.0 (8 female and 8 male) who stopped natalizumab treatment after a median number of 20 infusions (range 12-114). The demographic and clinical characteristics of patients are shown in Table 1.

Inclusion criteria were: age $\geq 18$ years, treatment with natalizumab for at least one year, positive JCV index, no evidence of clinical and radiological activity in the last 6 months of natalizumab treatment, contrast-enhancement brain MRI performed just before alemtuzumab administration without any sign of PML, and an aggressive disease course before natalizumab therapy. Exclusion criteria were: pregnancy or breastfeeding, clinically significant or unstable medical or surgical conditions able to preclude safe and complete study participation.

The decision to stop natalizumab was taken after a consultation between the neurologist and patient, in which the risks and benefits of continuing or interrupting natalizumab were explained. For all the patients, the reason for interrupting natalizumab was the high risk of PML. The switch to alemtuzumab was planned after a wash-out period of 2 months.

Patients underwent brain MRIs every three months during the natalizumab treatment and just before starting alemtuzumab in order to exclude any sign suggestive of PML. A contrast-enhancement brain MRI was then undertaken 6 and 12 months after the alemtuzumab infusion, in order to evaluate signs of disease reactivation (Fig. 1: study design). Brain MRIs were performed with the same 1.5 Tesla (T) scanner using the same protocol in each patient: the protocol included axial T2-weighted scans, coronal flair-weighted scans, DWI sequences and post-contrast T1-weighted scans.

\section{Compliance with Ethics Guidelines}

This article does not contain any new studies with human or animal subjects performed by any of the authors. 
Table 1 Clinical and demographic characteristics

\begin{tabular}{lllllll}
\hline Patients & Sex & Age & $\begin{array}{l}\mathbf{N}^{\circ} \\
\text { natalizumab } \\
\text { infusions }\end{array}$ & $\begin{array}{l}\text { EDSS at last } \\
\text { natalizumab } \\
\text { infusion }\end{array}$ & $\begin{array}{l}\text { Anti-JCV } \\
\text { index }\end{array}$ & $\begin{array}{l}\text { Previous } \\
\text { IS }\end{array}$ \\
\hline Pt 1 & M & 37 & 52 & 1.5 & 1.1 & No \\
Pt 2 & M & 36 & 114 & 2.5 & 1.2 & No \\
Pt 3 & M & 26 & 19 & 1.5 & 1.5 & Yes \\
Pt 4 & F & 34 & 62 & 2.0 & 3.5 & Yes \\
Pt 5 & F & 57 & 91 & 3.5 & 2.3 & Yes \\
Pt 6 & F & 24 & 20 & 1.0 & 1.1 & No \\
Pt 7 & F & 26 & 85 & 6.5 & 0.4 & No \\
Pt 8 & F & 20 & 15 & 1.0 & 0.8 & No \\
Pt 9 & M & 46 & 19 & 3.0 & 2.0 & Yes \\
Pt 10 & M & 38 & 39 & 2.0 & 1.1 & No \\
Pt 11 & M & 44 & 12 & 1.5 & 3.5 & No \\
Pt 12 & F & 46 & 12 & 2.5 & 2.3 & No \\
Pt 13 & M & 22 & 12 & 2.0 & 3.1 & No \\
Pt 14 & F & 41 & 84 & 4.0 & 1.1 & Yes \\
Pt 15 & M & 48 & 18 & 5.0 & 2.3 & No \\
Pt 16 & F & 41 & 13 & 2.5 & 2.8 & \\
\hline IS & & & & & \\
\hline
\end{tabular}

$I S$ immunosuppressive therapy

A Routinaly blood sample

Clinical evaluation

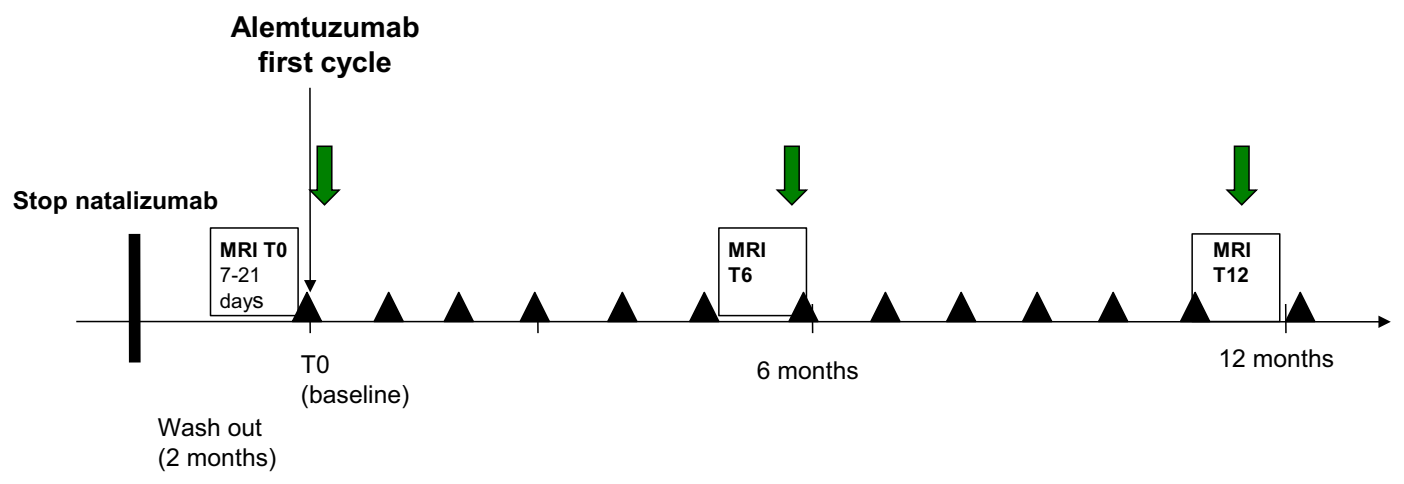

Fig. 1 Study design 


\section{RESULTS}

The median wash-out period from the last natalizumab infusion to starting alemtuzumab was 70 days (range 41-99): in this period, no relapse was observed. Furthermore, brain MRI performed just before starting alemtuzumab was unchanged compared to the previous one.

The first patient was treated with alemtuzumab on September 2015 and the last one on October 2016 and the range of follow-up is 7 days-12.6 months; at present, 8 out of 16 patients have a follow-up $>6$ months and 2 out of 8 reached 1-year follow-up; 5 have a follow-up of 3-6 months and 3 have a follow-up $<3$ months.

The brain MRI undertaken 6 months after alemtuzumab is available for 8 out of 16 patients and in all of them, neither signs of disease activity nor new lesions are present; in 2 out of 8 patients, the brain MRI at 12 months is also available, showing no sign of disease activity.

Clinical evaluation performed at 6 months and at 12 months showed stability, in particular, neither relapses nor increase in EDSS were observed.

\section{DISCUSSION}

The therapeutic landscape of multiple sclerosis (MS) is dramatically changing and becoming more and more complex for many reasons: (1) very early treatment is highly recommended [3-7]; (2) the number of therapeutic options has increased and the therapeutic algorithm is not fully established [2]; (3) very effective drugs are available; (4) tolerance toward the disease activity has been reduced, with the introduction of the criteria of no evidence of disease activity (NEDA) [8]; (5) neurologists have to face not only disease control but also the potential side-effects of new drugs.
Natalizumab represents an example of this changing scenario; it is a very effective drug, used to treat patients with an aggressive disease. The positive impact of the drug is clearly evident on many parameters: annualized relapse rate, EDSS, number of new lesions or enhancing lesions, quality of life [9-12]. Its limitations are the risk of PML and the therapeutic management of patients after drug interruption. To address the former limitation, neurologists can now stratify patients' risk of PML according to the JCV index, previous immunosuppressive therapy and duration of natalizumab exposure [13].

To address the second limitation, it is well-known that disease recurs after withdrawing natalizumab [14-18] and in order to limit this problem, the literature recommends to start a new treatment very early after natalizumab interruption [14-16]. However, which treatment is the most suitable is still a matter of great debate, as no guidelines exist about patients' therapeutic management. Therefore, the decision on how to treat and when to start treatment mainly derives from the neurologist's own experience and the characteristics of the individual MS patient.

Many studies about switching from natalizumab to other DMDs (interferons, glatiramer acetate, methylprednisolone) have been published showing disease reactivation [19-22]. Studies about switching from natalizumab to fingolimod showed a lower risk of recurrence of disease activity and that a shorter wash-out period ( $<12$ weeks) predicted a minor risk of disease reactivation [23, 24].

A recent study by Alping et al. [25] describes a population of 256 patients who shifted from natalizumab to fingolimod (55\% of cases) or to rituximab ( $45 \%$ of cases) due to the risk of PML. The study shows that within 1.5 year from 
natalizumab cessation, $1.8 \%$ of rituximab patients experienced a clinical relapse versus $17.6 \%$ of fingolimod-treated patients and that $1.4 \%$ of the rituximab group had contrast-enhancing lesions versus $24.2 \%$ of the fingolimod group.

We observed a smaller population of 10 patients who shifted to rituximab after a wash-out period of 2 months since interrupting natalizumab. We observed no clinical relapses nor contrast-enhancing or new lesions in any of the patients during the 1-year follow-up period [26].

The use of rituximab in patients with high risk of PML can be criticized; however, in rituximab-treated patients, PML was only observed in patients previously treated with immunosuppressive therapies for lymphoproliferative disorders, systemic lupus erythematosus or rheumatoid arthritis, and in no MS-affected patient [27].

In this study, we used alemtuzumab after natalizumab interruption. Patients in our study share some common characteristics: a very aggressive disease course before starting natalizumab, a high risk of PML, a stable phase of the disease during the natalizumab therapy and a very high quality of life. We had to take into consideration the need to treat a very severe disease and the safety profile of the drug to which we were switching, as well as to balance the risk of using aggressive drugs with the risk of a recurrence of disease activity if using less aggressive treatments.

Alemtuzumab is a very effective drug used successfully in patients with highly aggressive disease $[28,29]$ and the main side effect is the risk of autoimmunity [30]. Based on these data, we decided to use alemtuzumab and to start it 2 months after the last natalizumab infusion.

It is known that PML can occur a few months after withdrawing natalizumab, and that carryover PML can develop when patients have stopped natalizumab and already started a new DMT [31]. A recent study by Raisch et al. [32] reports cases of PML associated with biological therapies; a case of PML during alemtuzumab has been reported in a patient previously treated with natalizumab. As far as we know, in this patient, the symptoms of PML were already present when alemtuzumab was started but they were considered symptoms a relapse.

As alemtuzumab induces long-term immunosuppression with delayed immune reconstitution, if carryover PML develops after the alemtuzumab treatment, JCV cannot be counteracted by $\mathrm{T}$ cells. For this reason, some authors suggest the use of alemtuzumab after a bridging period of 6-12 months of oral treatment with fingolimod, dymethylfumarate or teriflunomide and to test JCV PCR in the CSF of seropositive patients before switching to alemtuzumab [33]. Others recommend delaying the start of alemtuzumab after a wash-out period from natalizumab of 6 months [34].

In our opinion, switching patients with aggressive disease to lower-efficacy drugs after interrupting natalizumab exposes them to too high risk of disease reactivation, as well as delaying alemtuzumab therapy by 6 months after the interruption of natalizumab. Furthermore, switching to fingolimod does not exclude the risk of carryover PML [31], in addition to exposing patients with aggressive MS to the risk of disease reoccurrence.

Regarding the detection of JCV PCR in CSF, it is known that a negative CSF does not exclude PML, and that in the early stage of PML, JCV may not be evident in CSF because the viral load is below the sensitivity of the virus assay. Furthermore, if radiological findings suggest PML, CSF may be negative [35]. In our study, 
we choose not to perform JCV PCR detection in the CSF before switching to alemtuzumab because of the risk of false negative. However, to minimize the risk of carryover PML, a strict radiological monitoring was planned through brain MRI performed every 3 months during natalizumab and just before starting alemtuzumab. Obviously, patients must be well-informed about risks and advantages derived from interrupting natalizumab and switching to alemtuzumab, and the decision to stop natalizumab and start alemtuzumab must be completely shared between the patient and neurologist.

\section{CONCLUSION}

Our study is ongoing and the results are only partial, but initial data are very promising as no patient had radiological signs or clinical symptoms of disease reactivation/progression, no patient experienced severe side effects and no patient developed PML.

Even though our population is small, no other data about the use of alemtuzumab after natalizumab have been described in the literature until now, except for a poster presentation [36], where the authors describe 13 patients shifted from natalizumab to alemtuzumab because of inadequate disease control. We think it is important to share our experience as the process of decision-making in therapeutic choices is very complex and data from "real world" can increase experience and knowledge of the whole neurological community.

\section{ACKNOWLEDGEMENTS}

No funding or sponsorship was received for this study or publication of this article. All named authors meet the International Committee of Medical Journal Editors (ICMJE) criteria for authorship for this manuscript, take responsibility for the integrity of the work as a whole, and have given final approval for the version to be published.

Design and supervision of the study: $\mathrm{AB}$. Clinical managements of patients: $\mathrm{MM}, \mathrm{MC}$, MLR, MM, FS, AdS; Wrote the paper: SM.

Disclosures. Simona Malucchi has received speaking honoraria and/or consultant fees from Biogen, Merck Serono, Novartis, Teva, and Genzyme. Marco Capobianco has received speaking honoraria and/or consultant fees from Biogen, Merck Serono, Novartis, Teva, Genzyme, and Almirall. Marianna Lo Re has received travel expenses from Biogen, Novartis, and Teva. Maria Malentacchi has received speaking honoraria from Biogen. Alessia di Sapio has received speaking honoraria and/or consultant fees from Biogen, Merck Serono, and Novartis. Manuela Matta has received speaking honoraria from Biogen. Francesca Sperli has received speaking honoraria from Biogen. Antonio Bertolotto has received honoraria for serving in the scientific advisory boards of Almirall, Bayer, Biogen, and Genzyme with approval by the Director of AOU San Luigi University Hospital, and has received speaker honoraria from Biogen, Genzyme, Novartis, and Teva; his institution has received grant support from Bayer, Biogen, Merck, Novartis, and Teva from the Italian Multiple Sclerosis Society, Fondazione Ricerca Biomedica ONLUS, and San Luigi ONLUS.

Compliance with Ethics Guidelines. This article does not contain any new studies with human or animal subjects performed by any of the authors. 
Data Availability. The datasets during and/ or analysed during the current study are available from the corresponding author on reasonable request.

Open Access. This article is distributed under the terms of the Creative Commons Attribution-NonCommercial 4.0 International License (http://creativecommons.org/licenses/ by-nc/4.0/), which permits any noncommercial use, distribution, and reproduction in any medium, provided you give appropriate credit to the original author(s) and the source, provide a link to the Creative Commons license, and indicate if changes were made.

\section{REFERENCES}

1. Coles AJ, Cox A, Le Page E, Jones J, et al. The window of therapeutic opportunity in multiple sclerosis: evidence from monoclonal antibody therapy. J Neurol. 2006;253:98-108.

2. Ziemssen T, Derfuss T, Stefano N, Giovannoni G et al. Optimizing treatment success in multiple sclerosis. J Neurol 2016;263(6):1053-65.

3. Prisms Study Group and University of British Columbia MS/MRI Analysis Group. PRISMS-4: long-term efficacy of interferon-beta-1a in relapsing MS. Neurol 2001;56:1628-1636.

4. Johnson KP, Ford CC, Lisak RP, Wolinsky JS. Neurologic consequence of delaying glatiramer acetate therapy for multiple sclerosis: 8-year data. Acta Neurol Scand. 2005;111:42-7.

5. Kappos L, Traboulsee A, Constantinescu C, Eralinna $\mathrm{JP}$, Forrestal $\mathrm{F}$, Jongen $\mathrm{P}$, et al. Long-term subcutaneous interferon beta-1a therapy in patients with relapsing-remitting MS. Neurol. 2006;67:944-53.

6. Kappos L, O'Connor P, Radue EW, Polman C, Hohlfeld R, Selmaj K, et al. Long-term effects of fingolimod in multiple sclerosis: the randomized FREEDOMS extension trial. Neurol. 2015;84:1582-91.

7. Goodin DS, Reder AT, Ebers GC, Cutter G, Kremenchutzky M, Oger J, et al. Survival in MS: a randomized cohort study 21 years after the start of the pivotal IFNbeta-1b trial. Neurol. 2012;78:1315-22.

8. Kappos L, De Stefano N, Freedman MS, Cree BA, et al. Inclusion of brain volume loss in a revised measure of 'no evidence of disease activity' (NEDA-4) in relapsing-remitting multiple sclerosis. Mult Scler. 2016;22(10):1297-305.

9. Kappos L, Belachew S, Butzkueven $\mathrm{H}$, et al. Long-term safety and efficacy and association between baseline treatment history and post-baseline relapses in multiple sclerosis patients treated with natalizumab in the TYSABRI Observational Program (TOP). Neurol. 2012;78(Suppl):1.

10. Hanna J, Rosen JP, Smethurst C. Efficacy and safety of treatment with natalizumab for relapsing-remitting multiple sclerosis: interim results of the Tysabri Observational Programme in the UK. J Neurol Neurosurg Psychiatry. 2013;84:e2.

11. Stephenson JJ, Kern DM, Agarwal SS, et al. Impact of natalizumab on patient-reported outcomes in multiple sclerosis: a longitudinal study. Health Qual Life Outcomes. 2012;10:155.

12. Svenningsson A, Falk E, Celius EG, et al. Natalizumab treatment reduces fatigue in multiple sclerosis. Results from the TYNERGY trial; a study in the real life setting. PLoS One. 2013;8(3):e58643.

13. Plavina T, Subramanyam M, Bloomgren $G$, et al. Anti-JC virus antibody levels in serum or plasma further define risk of natalizumab-associated progressive multifocal leukoencephalopathy. Ann Neurol. 2014;76:802-12.

14. Fox RJ, Cree BA, De Seze J, et al. MS disease activity in RESTORE: a randomized 24-week natalizumab treatment interruption study. Neurol. 2014;82:1491-8.

15. O'Connor PW, Goodman A, Kappos L, et al. Disease activity return during natalizumab treatment interruption in patients with multiple sclerosis. Neurol. 2011;76:1858-65.

16. Berger JR, Centonze D, Comi G, et al. Considerations on discontinuing natalizumab for the treatment of multiple sclerosis. Ann Neurol. 2010;68(3):409-10.

17. Sangalli F, Moiola L, Ferrè L, et al. Long-term management of natalizumab discontinuation in a large monocentric cohort of multiple sclerosis patients. Mult Scler Relat Disord. 2014;3:520-6.

18. Killestein J, Vennegoor A, Strijbis EM, et al. Natalizumab drug holiday in multiple sclerosis: poorly tolerated. Ann Neurol. 2010;68:392-5. 
19. Cohen M, Maillart E, Tourbah A, et al. Switching from natalizumab to fingolimod in multiple sclerosis: a french prospective study. JAMA Neurol. 2014;71:436-41.

20. Magraner MJ, Coret F, Navarre A, et al. Pulsed steroids followed by glatiramer acetate to prevent inflammatory activity after cessation of natalizumab therapy: a prospective, 6-month observational study. J Neurol. 2011;258:1805-11.

21. Havla J, Gerdes LA, Meinl I, et al. De-escalation from natalizumab in multiple sclerosis: recurrence of disease activity despite switching to glatiramer acetate. J Neurol. 2011;9(258):1665-9.

22. Lo Re M, Capobianco M, Ragonese P, Realmuto S, et al. Natalizumab discontinuation and treatment strategies in patients with multiple sclerosis (MS): a retrospective study from two Italian MS centers. Neurol Ther. 2015;4(2):147-57.

23. Iaffaldano P, Lucisano G, Pozzili C, Brescia Morra V, et al. Fingolimod versus interferon beta/glatiramer acetate after natalizumab suspension in multiple sclerosis. Brain. 2015;138(Pt11):3275-86.

24. Kappos L, Radue EW, Comi G, TOFINGO study group. Switching from natalizumab to fingolimod: a randomized, placebo-controlled study in RRMS. Neurology. 2015;85:29-40.

25. Alping P, Frisell T, Novakova L, Jakobsson PI et al. Rituximab versus fingolimod after natalizumab in multiple sclerosis patients. Ann Neurol 2016;79:950-58.

26. Malucchi S, Capobianco M, di Sapio A, Lo Re M et al. Rituximab suppresses disease activity after natalizumab withdrawal: an exploratory study. MSDD 2016;1-11. doi:10.1186/s40893-016-0013-z.

27. Henegar CE, Eudy AM, Kharat V, Hill DD, Bennett D, Haight B. Progressive multifocal leukoencephalopathy in patients with systemic lupus erythematosus: a systematic literature review. Lupus. 2016;25(6):617-26.

28. Hirst CL, Pace A, Pickersqill TP, Jones R. Campath $1-\mathrm{H}$ treatment in patients with aggressive relapsing remitting multiple sclerosis. J Neurol. 2008;255(2):231-8.

29. Le Page E, Deburghgraeve V, Lester MA, Cardiet I, et al. Alemtuzumab as rescue therapy in a cohort of 16 aggressive multiple sclerosis patients previously treated by Mitoxantrone: an observational study. J Neurol. 2015;262(4):1024-34.

30. Dubey D, Cano CA, Stüve O. Update on monitoring and adverse effects of approved second-generation disease-modifying therapies in relapsing forms of multiple sclerosis. Curr Opin Neurol. 2016;29(3):278-85.

31. Putzy N, Clifford DB, Bischof D et al. Characteristics of PML cases in multiple sclerosis patients switching to fingolimod from natalizumab. Presentation at ECTRIMS 2014.

32. Raisch DW, Rafi JA, Chen C, Bennett L. Detection of cases of progressive multifocal leukoencephalopathy associated with new biologicals and tergeted cancer therapies from the FDA's adverse event reporting system. Expert Opin Drug Saf. 2016;15(8):1003-11.

33. Giovannoni G, Marta M, Davis A, Turner B, et al. Switching patients at high risk of PML from natalizumab to another disease-modifying therapy. Pract Neurol. 2016;16:389-93.

34. Hassoun L, Eisele J, Thomas K, Ziemssen T. Hands on Alemtuzumab-experience from clinical practice: whom and how to treat. MSDD. 2016;1:10.

35. McGuigan C, Craner M, Guadagno J, et al. Stratification and monitoring of natalizumab-associated progressive multifocal leukoencephalopathy risk: recommendation from an expert group. J Neurol Neurosurg Psychiatry. 2016;87:117-25.

36. Fortin J, Jacques FH, Fokko VS, Grand'Maison F, Emond F, Bakdache F. Switching from natalizumab to alemtuzumab in patients with RRMS: real-world experience. ECTRIMS Mult Scler J. 2016;22(S3):783. 